\title{
ACTORES Y CONFLICTOS TERRITORIALES EN UNA FIGURA DE ADMINISTRACIÓN PÚBLICA DE LA PESCA ARTESANAL. EL CASO DE LA ZONA CONTIGUA EN LAS REGIONES DE LOS LAGOS Y DE AYSÉN, SUR DE CHILE ${ }^{1}$
}

CATALINA ÁLVAREZ B. ${ }^{a}$, CLAUDIO GAJARDO C..$^{\text { } ~ \& ~}$ FRANCISCO THER R.

\section{RESUMEN}

Durante siglos han existido desplazamientos de habitantes entre la Isla Grande de Chiloé, sus alrededores y el Archipiélago de las Guaitecas. Con posterioridad a la regionalización del país, los trayectos de pescadores de la Región de Los Lagos hacia Aysén debieron gestionarse bajo una figura política y administrativa. En este caso, la Zona Contigua implicó que los gobiernos regionales acordaran compromisos y compensaciones a través de Mesa público-privada encargada de definir con criterio político, científico, empresarial y pesquero-artesanal, los tipos y volúmenes de extracción de recursos. El presente trabajo describe las relaciones y estrategias que se despliegan en torno a la Zona Contigua y su Mesa público-privada, identificando actores y conflictos según contextos discursivos. La capacidad de agencia en los discursos de los actores da cuenta de un vínculo entre los planos sociopolítico y socioterritorial que, constituyendo una interfaz social, evidencian las oposiciones y/o complementariedades en las relaciones entre los actores y sus discursos. En su conjunto, se proveen de bases para una reflexión tendiente a preocuparse por evidenciar alternativas bajo una visión política integral y actualizada de la pesca artesanal.

PALABRAS CLAVE: sociedades litorales, actores y conflictos territoriales, antropología política, recursos pesquero-artesanales, pesca artesanal.

\section{ACTORS AND TERRITORIAL CONFLICTS IN A FIGURE OF PUBLIC ADMINISTRATION OF ARTISANAL-FISHERIES. IN THE CASE OF CONTIGUOUS ZONE IN LOS LAGOS AND AYSÉN REGION, SOUTH OF CHILE}

\footnotetext{
ABSTRACT

During centuries there has been population displacement between the surroundings of Isla Grande de Chiloé and the Guaitecas's Archipelago. After the regionalization of the country, fisherman's journeys

a Antropóloga. Programa ATLAS, Universidad de Los Lagos. catalinalvarez@gmail.com

b Antropólogo. Programa ATLAS, Universidad de Los Lagos. clgajardocortes@gmail.com

c Antropólogo. Programa ATLAS, Universidad de Los Lagos. Dirección Postal: Avenida Fuchslocher 1305, Campus Osorno, Casilla 933 - Osorno, Chile. Fono: +56+64+33 31 39. fther@ulagos.cl Proyecto 81100002

1 Valoración Económico-Ambiental de las Actividades Pesqueras Artesanales. Estrategias de Diversificación Productiva en Sociedades Litorales del Sur de Chile. Programa MEL - CONICYT - Chile.
} 
of Los Lagos Region to Aysén were managed under a political and administrative figure. In this case, the Contiguous Zone implied that the regional government agreed commitments and compensation through a Public-Private Table responsible of defining with political, scientific, business and artisanal-fisheries criteria, types and volumes of resource extraction. This work describes relationships and strategies that unfold around the Contiguous Zone and Public-Private Table, identifying actors and conflicts according to discursive contexts. The agency capacity in the speeches of actors shows a link between the sociopolitical and socioterritorial plans that, constituting a social interface, shows the oppositions and/ or complementarities in relations between the actors and their speeches. As a whole, basis for reflection intended to create an awareness about alternatives under a comprehensive and updated policy vision of artisanal fisheries are provided.

KEY WORDS: littoral societies, actors and territorial conflicts, political anthropology, recursos pesquero-artesanales, pesca artesanal.

\section{INTRODUCCIÓN}

La crisis ambiental implícita en los actuales modelos económicos está asociada a la apertura desregulada de recursos naturales en el mercado internacional (Schaper, 1995; Leff, 1996). En este contexto la debilidad en el manejo y administración de los recursos naturales remite tanto a la falta de administraciones articuladas como a la debilidad de las políticas públicas para integrar a los actores territoriales (Pamplona, 2000; Bárcena et al. 2012). En los ambientes marinos las políticas se han elaborado ya sea por departamentos, fragmentando la concepción de los espacios de uso (Andrade et al. 2008), o bien excluyendo a sus actores relevantes (Alcalá, 1999, 2003; Baliero et al. 2006). Las concepciones compartimentalizadas subyacentes a las políticas tienden a obstaculizar la visión integral, dificultando la distribución de responsabilidades en torno a la explotación de los recursos naturales (CEPAL/PNUMA, 2012). El presente trabajo aborda una política de administración de recursos pesquero-artesanales a través del estudio de sus actores y relaciones. En buena medida, el estudio de la Zona Contigua y de su Mesa Pública-privada, en tanto proceso sociopolítico de la pesca artesanal, es un estudio sobre el conflicto. Siguiendo a Martínez-Alier (2004, 2011), los conflictos territoriales acontecen al menos por tres situaciones: a) o suceden por una discrepancia de valoración dentro de un único sistema de valoración; b) en otros casos se pueden observar múltiples valores, existiendo una disputa sobre el propio sistema de valoración que debe ser empleado; c) o bien existen choques de lenguajes de valoración territorial o identiraria, desplegados contra otras valoraciones como la monetaria (Martinez-Alier, 2004; MartinezAlier, 2011). Al mismo tiempo, diversos autores destacan tres formas que tendrían los gobiernos para planificar los territorios y las relaciones entre actores, la mentalidad del neoliberalismo, el localismo y ecocentrismo; las que en su despliegue generarían tensiones por la competencia o colaboración en el manejo de recursos naturales (Barber, 2010; Ghermandi et al. 2010; Larsen et al. 2011; Lockwood \& Davidson, 2010). Para mitigar los conflictos, algunos trabajos enfatizan en la necesidad de desarrollar la interfaz científiconormativa (Bremer \& Glavovic, 2013), con lo cual se tiende a superar tanto los conflictos que surgen de las diferencias en los objetivos planteados por actores diversos (Wasson et al. 2015) como la falta de obligaciones legales para su implementación (Bullimore, 2014). En específico, en un estudio reciente, Zhao et al. (2015) evidencia la necesidad de fortalecer los mecanismos de cooperación entre actores e instituciones a objeto de contrarrestar la debilidad de enfoques de administración territorial basados únicamente en el mercado o normas del mercado estandarizadas con metas racionales (Zhao et al. 2015). En definitiva, se requiere de miradas integrales y actuales en torno a los conflictos y las formas de disminuir sus impactos. En esta línea, y para el caso de los usos de los espacios marítimocosteros en el sur de Chile, se reconocen en las últimas décadas, un creciente número de estudios que destacan tanto los aspectos socioculturales (Andrade et al. 2008; Cordero, 2011; Gelcich et al. 2010; Guerra \& Skewes, 2008; Saavedra, 
2008, 2011; Skewes et al. 2012), como los relativos a la aplicación de cuerpos legales como son la Política Nacional de Usos del Borde Costero (1994), la Ley General de Pesca y Acuicultura (1990) y la Ley de Espacio Costero Marino de los Pueblos Originarios (2008) (De la Maza et al. 2012; Martínez, 2012). En estos estudios se establece que las normativas son implementadas "de arriba hacia abajo" (topdown), omitiendo la integración de las continuidades y discontinuidades socio-históricas en los territorios (Bugueño, 2011). En específico, las discusiones y avances en relación a la pesca artesanal han abordado temáticas tales como la orientación a la sustentabilidad en los cuerpos legales (Duhart, 2004), los cambios en las políticas de gestión (Arthur, 2005; Berkes et al. 2001; Olsson et al. 2004a), la visibilización de redes de múltiples actores (Crona et al. 2009; Marin \& Berkes, 2010; Marín et al. 2012) y el avance hacia la integración y participación en las figuras de administración de recursos naturales (Berkes, 2006; Berkes \& Carlsson, 2004; Cundill, 2010; Gelcich et al. 2008; Keene et al. 2002; Olsson et al. 2004b; entre otros).

En consecuencia, los estudios sobre los conflictos territoriales han girado su mirada desde los sistemas de poder en culturas aisladas a la exploración de "el lugar de la política" en las sociedades contemporáneas (Abelés, 1997). Más que como una propiedad dada, el poder se manifiesta así como relaciones expresadas en el ejercicio práctico, e históricamente situado, de normativas consensuadas y estratégias pragmáticas entre actores, instituciónes y sujetos (Foucault, 2001). Los contextos de sobreexplotación de recursos y pérdida de referentes tradicionales condicionan estrategias de superviviencia coexistentes a la mirada clásica de la política como convivencia (Abelés, 2008). En este sentido, estudios sobre conflictos territoriales abordan de nueva cuenta las relaciones sociedad-Estado (Hevia, 2009), la construcción social de sistemas políticos (De la Maza, 2009), los procesos de gobernanza y participación (Montecinos, 2007), las interrelaciones entre actores (Long, 2007), las alianzas público-privadas (Devlin \& Mogullansky, 2009) y la direccionalidad en la aplicación de políticas públicas (Barragán \& Wanderley, 2009). En el presente trabajo se presenta una mirada antropológica centrada en la construcción cotidiana de sistemas político-culturales (De la Maza, 2009, 2012), el enfoque hacia los actores (Long, 2007) y el abordaje de sus prácticas y discursos como manifestación y ejercicio de relaciones de poder (Foucault, 2001).

\section{MATERIAL Y MÉTODOS}

La metodología se centró en un análisis interactivo de los discursos y prácticas (Hevia, 2009) en torno a la Zona Contigua. Los discursos de los actores, los espacios de interacción y la implementación de la política constituyen las principales unidades de análisis. En concordancia, los procedimientos siguieron un diseño cualitativo no-experimental guiado por una conceptualización previa del objeto y problema de estudio (Torres \& Becerra, 2006).

En la etapa de revisión de fuentes secundarias se buscó perfilar la institucionalidad y los aspectos legales de la actividad pesquero-artesanal, siendo un importante insumo los trabajos de Moreno et al. (2007) y Molinet et al. (2007), sobre todo en lo que respecta al origen de la Mesa PúblicoPrivada. En consideración que el principal recurso de disputas correspondía al Erizo (Loxechinus albus), se revisó la prensa escrita y los informes emitidos por la Comisión de Manejo de Pesquerías Bentónicas (COMPEB). Ésta exploración permitió identificar hitos históricos en la conformación de la Zona Contigua, los actores referenciados y los temas tratados en la Mesa Público-Privada. Posteriormente, se diseñaron instrumentos de recolección de información para cada uno de los actores. En la etapa de entrevistas y conversaciones con expertos se buscó reconocer el rol en la Mesa Público-Privada, así como el reconocimiento de otros actores. Se participó en algunas de las sesiones de la Mesa Público-Privada de la Zona Contigua, aplicándose observación directa, complementada con entrevistas a algunos dirigentes de la pesca artesanal. La observación directa y entrevistas con dirigentes tuvo por objetivo distinguir y caracterizar a los actores, observar las dinámicas relacionales entre ellos, principalmente sus estrategias, e identificar los temas tratados y el modo en que los actores los desarrollaban. Durante la visita a las caletas de pescadores se realizaron entrevistas a 
actores relacionados directa e indirectamente a la Zona Contigua. Se buscó caracterizar a los actores comparando sus discursos con la información de prensa previamente recogida. A partir de los relatos de pescadores entrevistados se buscó identificar los cambios en el desarrollo de la figura administrativa. Para esto se indagó respecto a qué sucedía antes y después de la instalación de la Zona Contigua, el presente y las proyecciones futuras visualizadas para la misma. En la etapa de sistematización se elaboró una matriz de doble entrada considerando la información contextual sobre políticas públicas de pesca artesanal, así se identificaron las vinculaciones entre órganos administrativos y disposiciones legislativas, el desarrollo histórico de la Ley de Pesca y los modelos de desarrollo económicos. La información recogida a través de entrevistas con expertos y pescadores fue sistematizada en una matriz de discursos relacionándola con los hitos evidenciados en la conformación histórica de la Zona Contigua Los Lagos - Aysén. Se agregaron notas y relatos asociados a las estrategias y percepciones sobre el pasado, el presente y las proyecciones. Finalmente, la interpretación y presentación de resultados se retomaron tópicos acerca actores, estrategias y conflictos.

\section{ÁREA DE ESTUDIO}

El área de estudio corresponde al espacio comprendido entre $41^{\circ} 20^{\prime} \mathrm{S}^{2}$ en el sector de Chacao, norte de la Isla Grande de Chiloé, y $46^{\circ}$ 56' S en la Península de Taitao, sector sur de la Región de Aysén (Fig. 1). En el borde costero del área de estudio se distinguen caletas de pescadores con mayor demografía, son los puertos de desembarque de Calbuco, Dalcahue, Quellón, Puerto Cisnes y Melinka. También se distinguen asentamientos intermedios con mayor equipamiento y diversificación de actividades tales como El Manzano, Chonchi y Pichicolo. $\mathrm{Y}$ asentamientos asociados a pequeños pueblos costeros tales como Cucao, Achao, Apiao, Catruman, Llanchid, Repollal y Quicaví. Finalmente, se distinguen asentamientos rurales y aislados, tales como Curaco de Vélez, Pumillahue y San Juan.

Desde el Seno de Reloncaví, en las riberas de Maullín.

Corresponde al buceo hookah, donde el pescador se equipa con un traje de goma, aletas, cinturón con plomos,
Esta amplia zona incluye una diversidad de sistemas estuarinos, fiordos, islas y canales (Pickard, 1971; Silva et al. 1958, citados en Molinet et al. 2007). Además, corresponde a una de las cinco eco-regiones del país, con características de rica biodiversidad especialmente por invertebrados marinos, peces, aves y mamíferos (Hucke-Gaete et al. 2010). Posee una significativa riqueza patrimonial, con registros arqueológicos y patrimonios indígenas y de tradiciones chilotas. En el ámbito socioproductivo se destacan prácticas relacionadas con el campo y mar (Bravo, 2004) que expresan una práctica consuetudinaria de usos en el borde costero (Skewes et al. 2012).

Dentro de la Zona Contigua trabajan 1.673 buzos en 1.212 embarcaciones. En total hay 4.709 personas en actividades extractivas, 1.500 como intermediarios y transporte y 4.786 personas en actividades de procesamiento en industrias. Considerando además los empleos indirectos habría 16.493 personas trabajando dentro de la zona (Molinet et al. 2007). Las principales especies extraídas son la almeja (Venus antiqua), culengue (Gari solida), erizo (Loxechinus albus), cholga (Aulacomya ater), chorito (Mytilus chilensis), luga roja, (Gigartina skottsbergii), luga negra (Sarcothalia crispata), jaiba marmola (Cancer edwardsii), y macha (Mesodesma donacium). Asociado a éstas especies, los pescadores realizan jornadas diarias de extracción para luego vender su producción a intermediarios. Para las especies de almeja, culengue y macha son trayectos de dos horas de navegación donde mediante buceo ${ }^{3} \mathrm{o}$ recolección (macha) trabajan en la extracción por períodos de cuatro horas (Ibíd.). Para la extracción del erizo, los pescadores pasan la noche en embarcaciones o en ranchas transitorias (Barahona et al. 2003). Las embarcaciones pequeñas son remolcadas por lachas de transporte a los bancos naturales y realizan faenas diarias pernoctando en las cercanías. Durante la jornada, los buzos extraen los recursos y los van entregando a lanchas transportadoras que realizan viajes diarios a los puertos de desembarque (Molinet et al. 2007).

La extracción de erizo, luga roja y luga negra, se acompaña de lanchas extractoras. Éstas

mascarilla y regulador de aire. El buzo recibe el aire a través de una manguera conectada a un compresor ubicado en la embarcación. 


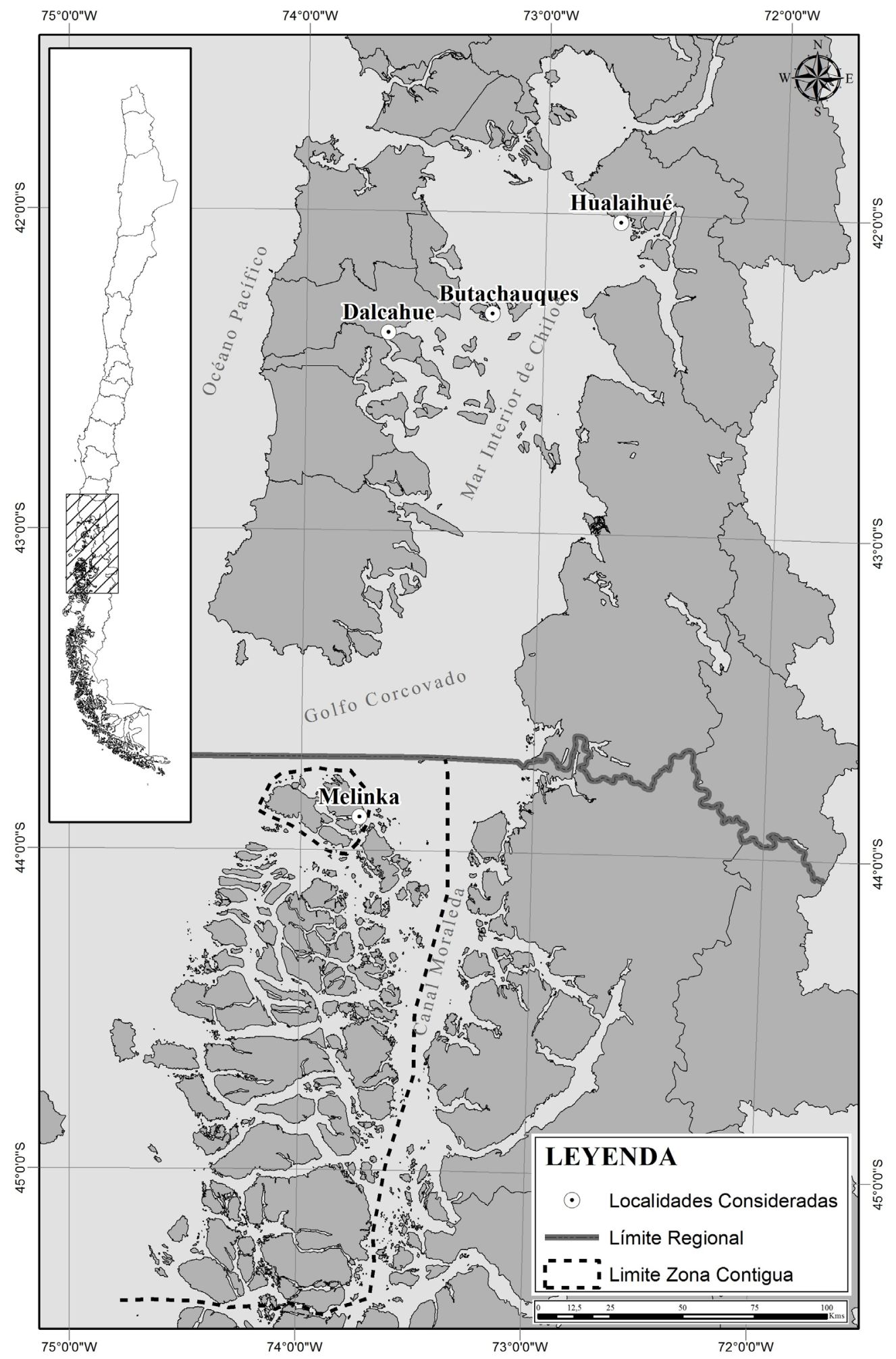

Fig. 1. Zona Contigua de la Región de Los Lagos y Aysén. (Diseño cartográfico: Zamir Bugueño). 
se encuentran equipadas con camarotes y cocina por lo cual permanecen por períodos superiores a un día cercanos a bancos naturales (Gutiérrez et al. 2006). En la recolección de algas se utilizan aparejos de enmalle que pueden alcanzar los 80 Kg. (Molinet et al. 2007). En el caso de la jaiba se identifican tres tipos de prácticas: a) jornadas diarias usando buceo y trampas; b) jornadas de más de un día en embarcaciones mayores; c) entrega a embarcaciones transportadoras hacia puertos de desembarque (Olguín et al. 2007). Las trampas son estructuras formadas por un enrejado de acero de forma cónica cubierta por una malla con un diámetro aproximadamente $100 \mathrm{~cm}$., en su interior se ubican carnadas de salmón, jurel, pejerrey y choritos (Molinet et al. 2007). Los individuos entran por la parte superior y quedan atrapados debido a que en la boca superior de la trampa hay un plástico que impide su agarre en la malla.

\section{RESULTADOS}

\section{Desplazamientos y apropiaciones}

históricas en la actual Zona Contigua

Los desplazamientos tradicionales de pueblos indígenas es el primer antecedente en la actual Zona Contigua. Estos desplazamientos remiten a los viajes y trayectorias de grupos indígenas entre el mar interior de Chiloé y las Islas Guaitecas, el archipiélago de los Chonos y más al sur. Efectivamente, distintos grupos indígenas de movilizaban desde los Archipiélagos de los Chonos y Guaitecas, e incluso desde más al sur, viajaban por el mar interior hasta llegar a la parte norte de la isla grande de Chiloé (HuckeGaete et al. 2008; Aspillaga et al. 2006; Álvarez, 2002; Legoupil, 2005; Emperaire, 1963). Con estos desplazamientos cotidianos se conformó un corredor marítimo prehispánico estableciendo un tránsito longitudinal en el espacio marino actualmente entre las regiones de Los Lagos y Aysén.

Un segundo hito histórico lo constituyen los viajes que las misiones jesuitas realizaron por este corredor. Para Legoupil (2005), la existencia temprana de cazadores-recolectores marítimos en las cercanías de Quellón ligados con los Chonos del Sur, explica "la ayuda eficaz de los indígenas de Chiloé a las expediciones marítimas jesuitas de los Padres Venegas y García, o de Juan Vicuña, en estas zonas alejadas, en el transcurso del siglo XVIII" (Legoupil, 2005, p. 55). En general, los viajes de embarcaciones realizados durante la colonia eran guiados por los "prácticos", indígenas con conocimiento sobre la geografía, el clima y las rutas de navegación (Bahamonde, 2006). Es probable que de alli derive una continuidad en las rutas que unen la isla grande de Chiloé a las Guaitecas y el Archipiélago de los Chonos, traspasadas por los indígenas a los Jesuitas y colonizadores. Los viajes de las misiones partían desde Dalcahue y las cercanías de Quellón con dirección a Melinka. Entre sus motivaciones estaba la de traer habitantes originarios a lugares más cercanos para continuar con el proceso de evangelización (Ther Ríos, 2011).

Posteriormente, y hasta entrar el siglo XX, se conforman poblados de pescadores artesanales en ambas regiones. La colonización de Aysén, da cuenta de un eje de poblamiento insular para las islas Guaitecas e islas Huichas, de tradición Huilliche y cuya tendencia productiva ha sido siempre bentónica. Además, un eje de poblamiento costero desde Piti-Palena hasta Puerto Aysén, caracterizado por actividades de pesca demersal (Martinic, 2005, citado en Molinet et al. 2008; Saavedra, 2011). En dicho proceso, se refuerza "la existencia de sistemas marítimos, desde la isla de Chiloé hasta zonas australes (...) como una zona de tránsito entre pueblos vecinos" (Hucke-Gaete et al. 2008, p. 22). Éstos poblamientos y la conformación de caletas y pueblos de pescadores conformó un modo de vida marítimo cuyo tiempo transcurre principalmente 'entre caletas' (Munizaga, 1988, p. 66); pero también, la apropiación del espacio marino se expresa en trayectos entre localidades terrestres $e$ insulares generando interconexiones socioespaciales duraderas en el tiempo.

En la Constitución de 1828 el país estaba dividido administrativamente en ocho provincias ${ }^{4}$ siendo Chiloé una de ellas. Posteriormente, en 1834 la Provincia de Chiloé se dividió en diez departamentos. En 1927 se establecieron los límites del departamento de Castro del modo 

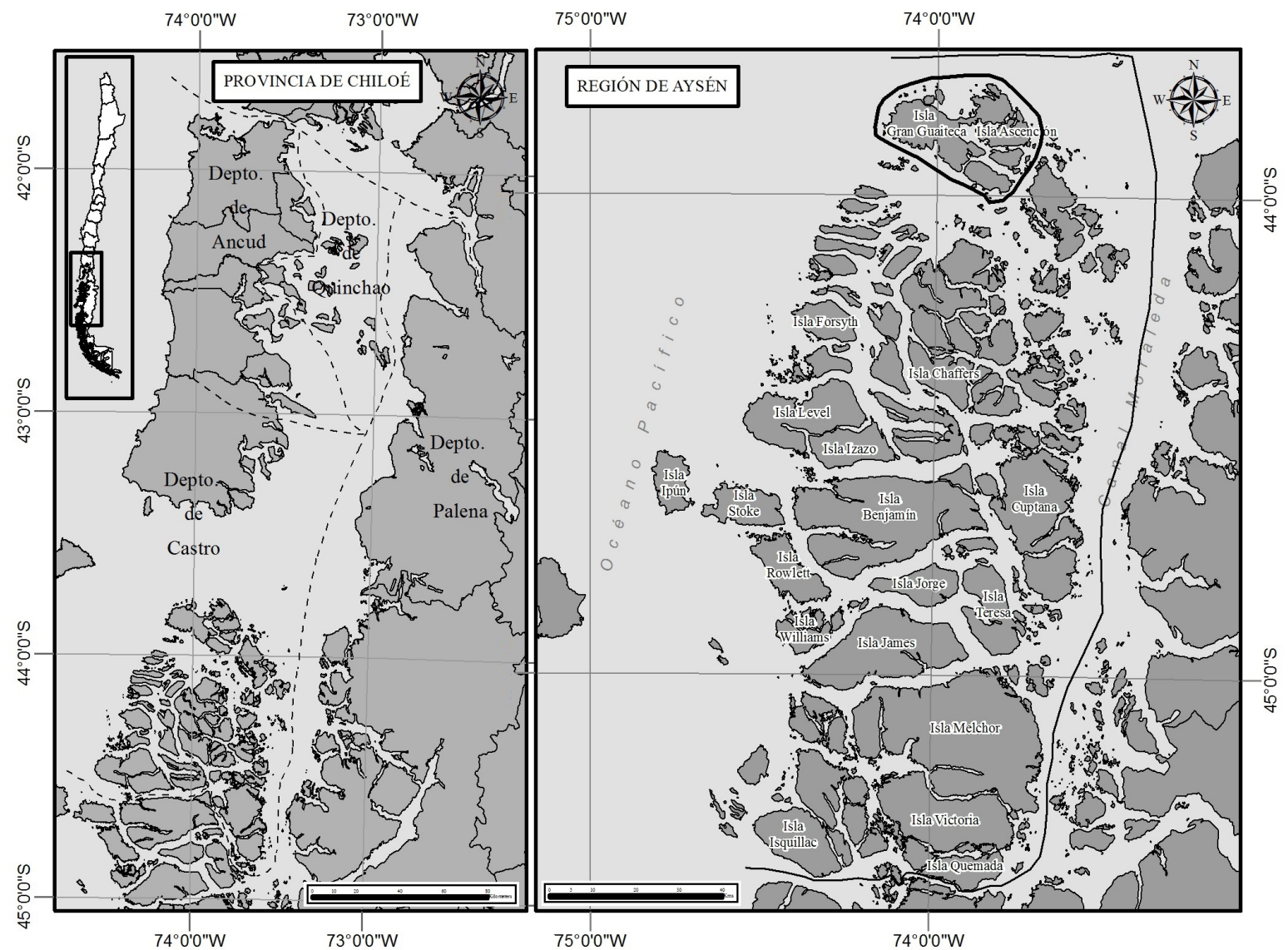

Fig. 2. Provincia de Chiloé, previo a la regionalización de 1974. (Diseño cartográfico: Zamir Bugueño).

siguiente:

El departamento de Castro estará formado por la parte de los actuales departamentos de Castro y Quinchao, que queda comprendida en la Isla Grande de Chiloé y los archipiélagos adyacentes, incluyendo la isla Chauque, el archipiélago de Quinchao, las islas Desertores, la de Guafo, y la parte del archipiélago de los Chonos, que queda al Poniente del Canal Moraleda y al Norte de los canales King y Pérez ${ }^{5}$

Luego de diversas fusiones y cambios, durante las modificaciones realizadas en 1959 correspondientes a la creación de los departamentos de Palena, Aysén, Coyhaique

5 El último decreto que establece los límites del departamento de Castro es el DFL 8582 de 1928. (Cursivas nuestras).

6 Ley N. ${ }^{\circ}$ 13.375. Crea y fija los límites de los departamentos y Chile Chico, la provincia de Chiloé queda dividida en los departamentos de Ancud, Castro, Quinchao y Palena ${ }^{6}$ (Martinic, 2005) (Fig. 2). Hasta estas últimas modificaciones el departamento de Castro aún incluía a las Islas Guaitecas, quedando integrados los vínculos que hasta el momento habían sido creados con la Isla Grande de Chiloé. En efecto, tras la migración de chilotes dedicados a las labores de explotación de Ciprés (Pilgerodendron uviferum), recolección de orilla, caza de lobos marinos y pesca artesanal, unidos por lazos parentales, costumbres, saberes y comercialización, se consolida un carácter identitario asociado a la población de Chiloé en el archipiélago de las Guaitecas (Nuñez, 2010; Hidalgo, 2011; Marticorena, 2009; Navarro, 2008).

de Palena, Aysén, Coyhaique y Chile Chico y las de sus respectivas comunas-subdelegaciones. Consultado el 21 de Diciembre de 2013. 
La actual división administrativa que establece las Regiones de Los Lagos y Aysén data de 1974. En ella, el sector de las Islas Guaitecas ${ }^{7}$ deja de pertenecer a la Región de Los Lagos pasando a la Región de Aysén. Esto implica una nueva distribución de recursos estatales y el énfasis por la colonización del territorio. Sin embargo, se hace evidente que éste corte tajante desconoce los usos tradicionales del territorio marítimo-costero, lo cual deriva en la conformación de una administración obstructiva a los lazos parentales y rutas de navegación y extracción previamente consolidados. Sin embargo, los pescadores artesanales de la Región de Los Lagos siguieron realizando sus faenas de pesca en las zonas del sector de Guaitecas, ahora pertenecientes a la Región de Aysén. Estos trayectos se mantuvieron sin mayores problemas, aun cuando la Ley General de Pesca y Acuicultura de 1991, estableció la regionalización y registro de pescadores (RPA), lo que implicaba que los pescadores pertenecientes a la Región de los Lagos no pudieran realizar prácticas extractivas en la Región de Aysén.

El período que va desde 1980 hasta el 2000, existieron muchas presiones sobre los pescadores de la Región de Aysén, emergiendo un conflicto con los pescadores que realizaban actividades en su sector. Entre ellas la aparición de marea roja en la Región de Aysén (Muñoz

\section{HITOS EN LA CONFORMACIÓN HISTÓRICA DE LA ZONA CONTIGUA LOS LAGOS - AYSÉN}

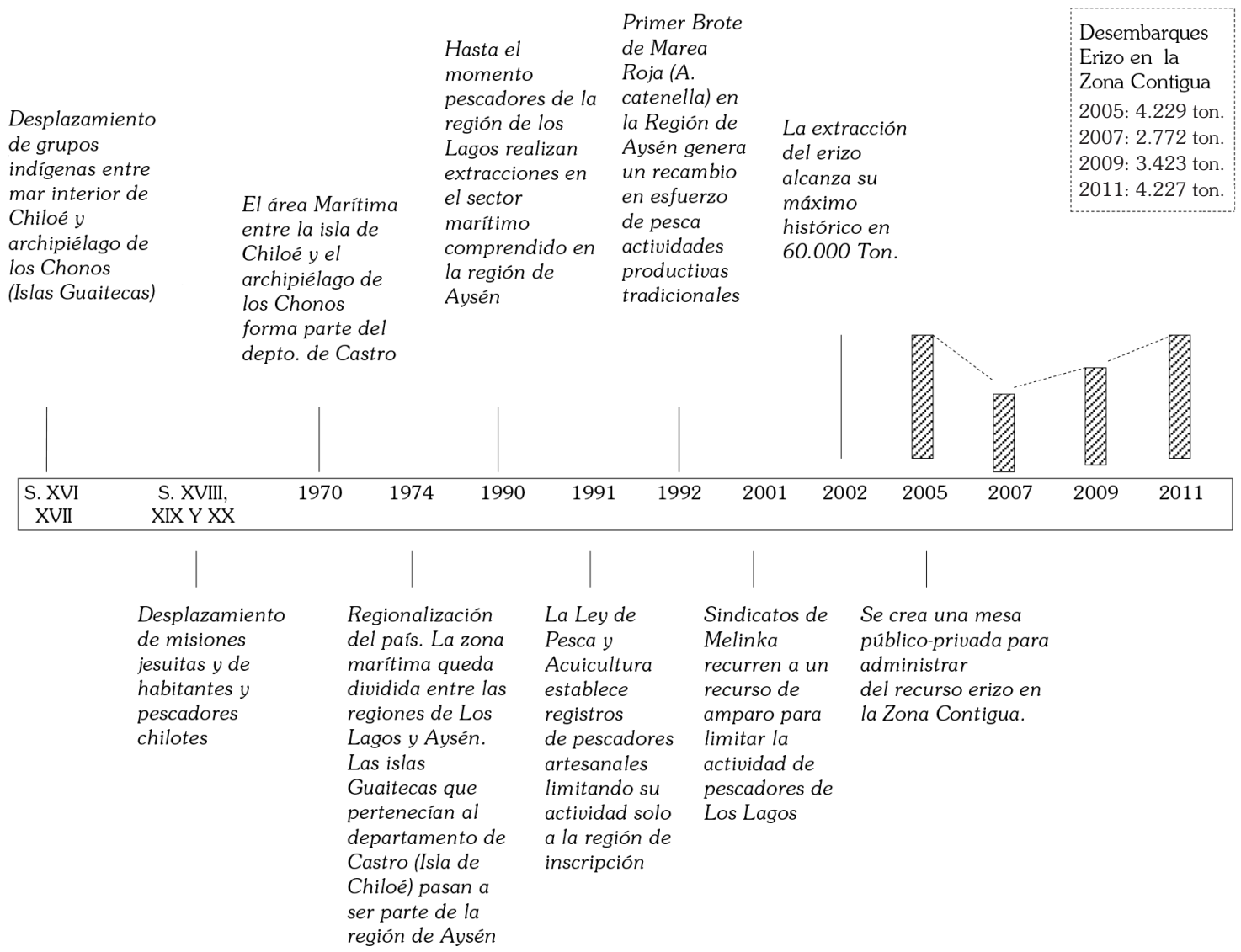

Fig. 3. Principales eventos que contribuyeron a la conformación tanto del conflicto entre los pescadores de Los Lagos y Aysén, como de la posterior conformación de la Zona Contigua y la Mesa Público-Privada (COMPEB). Las barras hacia el final de la figura muestran el inicio y la continuidad de la etapa científico-administrativa del proceso. Éstas tienen la información sobre los desembarques del recurso erizo administrado y delimitado por la Mesa público-privada para ésta especie. 
et al. 1992; Medina, 1997) que afectó a especies bentónicas tales como Chorito y Cholga, generaron una decaída de las prácticas tradicionales extracción y secado la Cholga. La marea roja también alcanzó la isla de Chiloé, específicamente en Quellón y otros sectores (Labra, 2011). En respuesta a dichos cambios, los pescadores se concentran en la extracción del erizo (Marticorena, 2009). Lo anterior, se ve agudizado por la creciente explotación del erizo que, iniciando su explotación en el período de 1930 a 1976 con 5.000 ton alcanza las 60.000 ton entre 1994 y el 2002 (IFOP, 2012).

En el año 2001 representantes sindicales de Caleta Melinka, con motivo de impedir el flujo extractivo de pescadores de la Región de Los Lagos, recurren a un recurso de amparo para limitar a esta acción abogando que dichas prácticas implicaban pérdidas económicas y ambientales. En respuesta, se genera una compensación económica ${ }^{8}$ de común acuerdo. En ella se permitiría la extracción y el libre tránsito entre ambas regiones según lo establecido en el artículo $\mathrm{N}^{\circ} 50$ de la Ley 18.892. En suma, este suceso se convierte en el punto decisivo a partir del cual se crea la Mesa Público-Privada. En el 2005 se crea un Plan de Manejo para el recurso Erizo en la Zona Contigua. Allí se definen las cuotas a extraer y se monitorea su cumplimiento (Fig. 3). Se establecen también las compensaciones a los pescadores del sector sur por la extracción que realizan pescadores del sector norte (Molinet et al. 2007). Hasta ahora, los pescadores de la región norte, especialmente de Chiloé, reclaman derechos históricos sobre el sector marítimo de la región sur (Saavedra,

8 La compensación económica, sólo es para los pescadores inscritos en el sindicato (el único a la fecha) de Caleta Melinka, lo que excluye a otras caletas y sindicatos de la región de Aysén, tema no exento de conflictos. La compensación es llevada a cabo mediante la inversión en equipos para el mejoramiento de la actividad.

9 La Mesa Público-Privada es itinerante, no tiene un lugar físico determinado para realizarse y tampoco fechas estipuladas anualmente. Todo depende de las circunstancias en que se encuentre el mercado, la producción y el ambiente social. Reuniones se han realizado en Puerto Montt y Coyhaique. Melinka o Quellón se ven como lejanos para hacerlas por la falta de infraestructura o por ser lugares conflictivos y con posibles movilizaciones sociales. El espacio usado es alrededor de una mesa donde todos pueden verse
2007). Mientras que éstos últimos abogan por cuidar el sector, especialmente el recurso erizo.

\section{Los actores territoriales}

en la Zona Contigua

Los actores que participan en la mesa de la Comisión de Manejo de Pesquerías Bentónicas (en adelante COMPEB) representan a ambas regiones, y corresponden a funcionarios públicos, políticos, representantes de pescadores artesanales, científicos y empresarios. ${ }^{9}$ Siguiendo un orden funcional a sus roles en la Mesa PúblicoPrivada, más que por su relación al conflicto (Marin \& Gelcich, 2012), se diferencian cinco actores territoriales, diversos internamente: a) el Estado; b) los pescadores artesanales; c) los científicos; d) las empresas; e) el orden público (Fig. 4). Los funcionarios públicos, sustentan su accionar en la institucionalidad, a partir de ahí despliegan prácticas político-administrativa hacia el resto de actores territoriales. Mientras existen funcionarios específicamente políticos, hay otros se mantienen en una esfera más bien de índole técnica. Por su parte los pescadores artesanales, a pesar de tener un pasado común en sus prácticas de apropiación del litoral, se diferencian principalmente por el territorio donde viven y extraen recursos. Su residencia en el sur de Chiloé o en el Archipiélago de las Guaitecas implica intereses disímiles. Los científicos se distinguen de profesionales asociados a consultoras ${ }^{10}$ correspondientes en su mayoría a biólogos marinos $e$ ingenieros acuícolas y a los asociados al sector académico correspondientes a ecólogos y biólogos marinos. Al igual que

las caras. Los temas de discusión son resolutivos. En la cabecera de la mesa se ubican las autoridades de gobierno de ambas regiones $\mathrm{y}$ los representantes institucionales del sector correspondientes de cada región. A un lado se encuentran los científicos y junto a ellos, también agrupados se encuentran los biólogos marinos que asesoran el plan de manejo. Al otro lado se ubican los empresarios y los encargados del transporte y su comercialización. En el extremo se encuentran los representantes sindicales de los sectores de Quellón y Melinka (diario de campo elaborado durante la visita a la mesa público-privada).

10 El Plan de Manejo de la Zona Contigua está sujeto a la asesoría de una consultora, que mediante evaluaciones permite evaluar el estado de disponibilidad de los recursos a extraer. Trabajan biólogos marinos fundamentalmente. 


\section{IDENTIFICACIÓN DE ACTORES Y DISCURSOS}

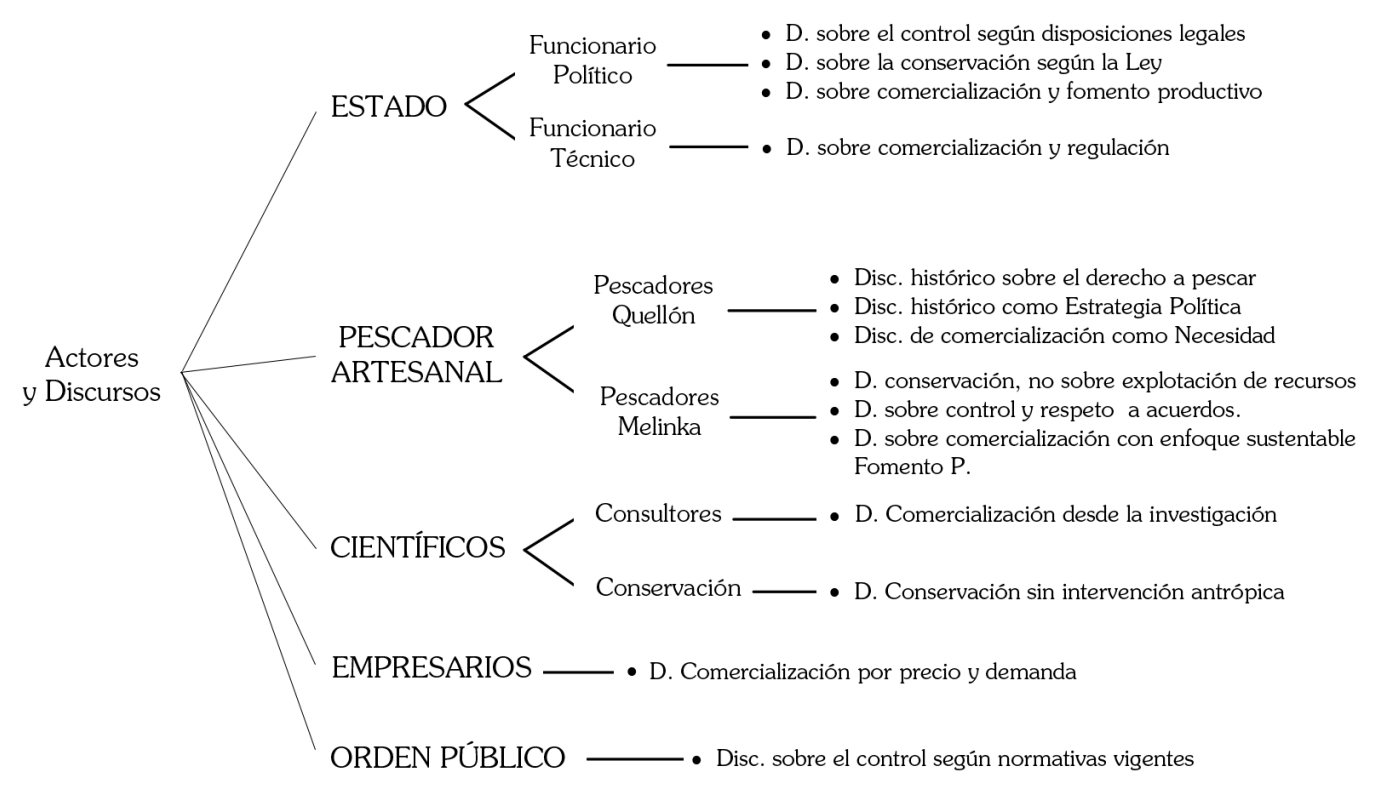

Fig. 4. Los actores no poseen un discurso unitario, están vinculados a las instancias en que los necesitan. Son parte de las estrategias que desarrollan en relación con otros actores y, por tanto, los discursos no tienen los mismos sentidos para la totalidad de actores. Cada uno de ellos los simboliza de manera distinta. Esto permite comprender lo que parece contradictorio, como que un actor este hablando de extracción minera y sustentabilidad al mismo tiempo.

los empresarios y los representantes de orden público, constituyen actores relativamente homogéneos.

\section{Relaciones entre actores}

Entre los funcionarios públicos, se distinguen funcionarios políticos que tienden a ser conciliadores de intereses estatales, desarrollando estrategias normalizadoras de las actividades de otros actores, a través de sus constantes intervenciones con discursos que incorporan dimensiones del ser y deber ser, definidas en las normativas. Los funcionarios técnicos, por su parte, aun cuando comulgan con los intereses estatales, experimentan cotidianamente las contradicciones de la aplicación de normativas por lo cual no se identifican sólo con su aplicación, sino que dan respuestas conciliadoras introduciendo adaptaciones a los contextos, situaciones y relaciones con los pescadores, sus dirigentes y territorios. En ambos casos, las acciones discursivas están orientadas a no cuestionar los actuales ordenamientos, instituciones y sus funcionamientos, sino a exigir que todo puedan ser observado desde el funcionamiento de lo público. Ejemplo de ello es una recurrencia constante al control técnico de las actividades de pesca, tanto en la costa como en el mar abierto, con el fin de eliminar los desembarques ilegales, la extracción de recursos no autorizados y el mercado negro.

En términos socioterritoriales, los pescadores artesanales poseen un origen común, de manera que sus miembros comparten lazos parentales, costumbres, mitos y saberes tradicionales, producto de constantes migraciones entre los Lagos y Aysén (Solari et al. 2012). Se componen, por un lado, de los representantes de pescadores miembros de Caleta Quellón inscritos en el RPA de la Región de Los Lagos. Su flota pesquera, de gran calado, es recurrentemente criticada por el otro grupo de pescadores aduciendo que dicha cualidad los excluye del sector propiamente artesanal. Por otro lado, se integran a este actor los representantes de pescadores miembros de Caleta Melinka, pertenecientes a la Comuna de Las Guaitecas e inscritos en el RPA de la Región de Aysén. Sus flotas son de menor calado en comparación a los pescadores de la Región de Los Lagos. Teniendo 
exclusividad de pesca en el polígono de exclusión ubicado alrededor de las Islas Guaitecas. Además reciben aportes económicos para sus equipos, como compensación por la extracción de recursos por parte del otro grupo de pescadores en su región.

Los científicos son observadores de "un ecosistema que sustentan procesos esenciales así como necesidades humanas", alejándose discursivamente de la visión asociada a recursos naturales, en tanto mercancías más o menos capaces de generar dinero (Berkes en Chirif, 2008, p. 11). Sus discursos reflejan posturas de conservación o de integración de instituciones y comunidades en torno a la administración de recursos naturales. Se compone de científicos académicos asociados a instituciones universitarias que están en contacto con pescadores artesanales por medio de investigaciones o consultorías. En estos acercamientos han ido priorizando la investigación sobre biodiversidad marina más que los vínculos posibles entre ésta y las sociedades de pescadores artesanales. También se asocia a este actor a las consultoras, organismos asesores, dispuestos por la ley para intervenir con el fin de apoyar el desarrollo y gestión económica de los pescadores artesanales. $\mathrm{Su}$ función es entregar los antecedentes técnico necesarios para fijan las cuotas de extracción para los pescadores. Sus discursos reflejan ciertas relaciones conflictivas con los pescadores quienes, al no compartir sus prácticas extractivas y su relación con la biodiversidad, impiden avanzar en el mejor conocimiento del área. Científicos y consultoras sostienen discursos diferenciados sobre la disponibilidad de recursos marinos para su comercialización o su conservación.

Los empresarios los integran tanto intermediarios como también la industria procesadora. Son quienes hacen presente en la Mesa Público-Privada la dimensión de la cadena productiva de la pesca artesanal, interesándose por temas que van desde la compra en la playa hasta las empresas procesadoras y distribuidoras. Los empresarios no poseen conflictos entre sí, más bien tienden a tensionar las relaciones entre los científicos y los pescadores artesanales, llegando a ser determinantes al momento de detonar el conflicto con las autoridades estatales. Ya que al presionar por el constante aumento en la cuota de las especies rentables, introducen fuerzas contrarias a la aplicación de vedas abogando por requerimientos para su comercialización. Finalmente, el actor "orden público" compuesto por las Fuerzas Armadas, es el actor más neutral. Su rol se limita a fiscalizar normativas establecidas por el Ministerio de Defensa Nacional que actúa por medio de la Subsecretaría para las Fuerzas Armadas. Esto lo hacen sin problematizar los usos de los espacios, sus actores o la disponibilidad y extracción de cuotas pesqueras. Son demandados por los pescadores artesanales para que ejerzan el cumplimiento de las normativas. Cuando las normativas tienen diversas interpretaciones, se observan ciertas presiones hacia las labores de este actor.

Los principales temas que son tratados en la Mesa Público-Privado se refieren al transporte de los recursos extraídos, los puntos de desembarque, la fiscalización de la marea roja, la evaluación en la disponibilidad de recursos y las condiciones sanitarias de los recursos extraídos. Estos temas hacen surgir situaciones algunas veces algo conflictivas entre los actores. Ante conflictos, los actores actúan en bloque o por separado, incluso formando nuevas alianzas. Una de las estrategias desarrolladas por el actor "Estado" es elaborar mapas sociales para orientar la normalización de la actividad pesquero-artesanal por medio de las normativas. En su consecución aparecen las funciones de los funcionarios públicos quienes estandarizan la actividad manteniendo contacto con dirigentes y representantes políticos. Por otra parte, los funcionarios técnicos dirigen sus esfuerzos a realizar este cometido mediante alianzas con otros actores, sobre todo durante actividades de terreno.

Por su parte, los pescadores artesanales con el tiempo han logrado especializar su lenguaje, conteniendo conocimiento técnico y administrativos. Su tendencia es a desarrollar relaciones estratégicas con los funcionarios públicos por sobre su interés con los científicos. Sin embargo, los pescadores de Caleta Quellón trabajan constantemente con biólogos marinos de consultoras, con quienes presionan para mantener los niveles de extracción de sus flotas. Por su parte los pescadores de Caleta Melinka tienden a buscar asesoramiento jurídico tanto para regular el acceso de otras flotas hacia Aysén. Ellos trabajan mediante 
coordinaciones locales $y$, en contraste con los pescadores de Quellón, mantienen cierta distancia el actor "científico". En términos generales, tienden a establecer relaciones estratégicas para participar en el sistema de mercado o apoyar la conservación. El principal objetivo de los pescadores artesanales en la Mesa Público-Privada es mantener su actividad y oficio.

Los científicos cumplen un rol general de asesoría a la gestión de los pescadores. A pesar de que dichos vínculos se predispongan en los cuerpos de ley, ellos asumen que las relaciones y alianzas toman tiempo antes de consolidarse. Los biólogos marinos asociados a consultoras y los ecólogos asociados a la academia poseen nociones y discursos conflictivos entre sí; contrastando, principalmente, sus discursos respecto a la disponibilidad de los recursos. Mientras las consultoras abogan por una amplia disponibilidad de recursos para las flotas pesqueras, los científicos argumentan que la disponibilidad de recursos se supone siempre como algo limitado.

\section{DISCUSIÓN}

Desde el punto de vista de su funcionamiento y de los actores participantes, la Zona Contigua ilustra que la noción de un Estado jerárquico y centralizado puede ser desplazada por la noción de co-construcción del Estado. En efecto, los discursos desarrollados por los actores dan cuenta de relaciones entre políticas de desarrollo, formas administrativas, legislaciones, funcionarios públicos, pescadores artesanales, empresarios y científicos (Gómez, 2003; De la Maza, 2012; Schavelzon, 2008). Como figura de administración política, la Zona Contigua congrega distintos significados. En primer lugar los significados centrados en la interacción y representación desde donde es vista la Zona Contigua como un espacio de diálogo entre actores ${ }^{11} \mathrm{o}$ una instancia democrática participativa. En segundo lugar, aquellos sigificados asociados a los conflictos desde donde se entiende a la Zona Contigua como una respuesta política frente a las demandas de los pescadores por la fijación de

11 Es necesario cuidar no caer en observaciones individuales, más aún cuando nos encontramos en situaciones conflictivas. Esta situación hace necesario triangular información tanto en lo observado, como por lo obtenido cuotas o bien como una estrategia que facilita el desarrollo de intereses económicos por sobre los ambientales. Y, en tercer lugar, desde el ejercicio del control y la normalización, la Zona Contigua se ve como una vía para formalizar las prácticas pesquero-artesanales y generar formas de control frente a posibles movilizaciones sociales.

Las relaciones entre los actores y sus formas de apropiación de diversos factores al interior de la Mesa Público-Privada asociada a la Zona Contigua, se contextualizan en la incertidumbre implícita en las instancias donde se logra la consecución de intereses. En general, cada actor en la Mesa Público-Privada evidencia dinámicas relacionales para el modelo de desarrollo de la pesca artesanal. Así, por ejemplo, si bien el mercado muestra un aspecto determinante, también recibe determinaciones externas tanto en las restricciones de acceso a pesquerías como en las cuotas de extracción recursos, contexto en el cual los científicos muestran una dimensión activa en la resolución y producción de relaciones entre pescadores, mercado y estado (Díaz, 2008). En este contexto, más que sus asociaciones transitorias, la pesca artesanal adquiere un posicionamiento tendiente a dar continuidad tanto a su oficio como a sus formas de trabajo.

Las transformaciones histórico-regionales vinculadas al conflicto entre pescadores artesanales permiten dar cuenta de ciertas formas de instanciación del oficio pesquero artesanal. Los pescadores remiten a "criterios constitutivos que recuperan a objeto de entrar en la disputa social" 12 . Tanto los pescadores de Quellón como los de Melinka son representados bajo la figura de pescadores artesanales ante la ley, las disparidades en sus discursos expresan la flexibilidad de dicha categoría. Aún con un origen territorial común, los pescadores de Melinka evocan criterios excluyentes asociados a la capacidad extractiva y al daño medioambiental que puede generar. En este sentido, las prácticas pesquero-artesanales y su vínculo con la biodiversidad marina, se territorializa mediante conflictos internos, claramente derivados de la apertura económica del país.

en entrevistas, informes técnicos o presa.

12 Universidad Austral (2011). Aldo Mascareño y Juan Carlos Skewes, debate en la UACh sobre Cultura [Video]. Disponible en: http://www.youtube.com/watch?v=t61W3hhmSh4. 
Resaltan en éste escenario el dinamismo y flexibilidad con que se manifiestan los contextos circunstanciales en que se desarrolla la Zona Contigua. Los discursos de los actores sobre un mismo aspecto normativo se encuentran fuertemente motivados por los intereses. A partir de lo anterior las interpretaciones divergen y la validez de las argumentaciones queda en segundo plano, dando paso al uso y apropiación de conocimientos para sustentar estrategias de acción, ya sea en busca de permanencia en ciertas áreas o de la emergencia de nuevas disputas dentro del conflicto. Este conjunto de significaciones dan cuenta de la Zona Contigua como una arena política dinámica que, en efecto, ha sido reconocida por sus miembros como una alternativa para la participación y el diálogo. La imagen evidenciada en sus relatos y relaciones da cuenta de aspectos relacionales más que de imposiciones definitivas, concepción que permite ir más allá de la visión tradicional de verticalidad en la elaboración de políticas públicas (Long, 2007).

\section{CONCLUSIONES}

La Zona Contigua surge en estrecho vínculo a factores históricos y culturales presentes en las prácticas de pescadores artesanales de las regiones de los Lagos y de Aysén. En este sentido, constituye una instancia de conformación diferente al normal surgimiento de una política de administración. Dicho carácter permite evidenciar el campo relacional de la pesca artesanal en términos de las dinámicas internas de ésta figura de administración.

En la antropología política y su relación con estudios sobre la pesca artesanal, el enfoque centrado en el actor permite abordar la diversidad y complejidad de instancias contemporáneas de participación y juego de intereses en la sociedad. Bajo ésta mirada los actores son reconocidos como agentes con la capacidad de apropiarse, transformar, negociar y resistir la influencia de factores exógenos y endógenos. De la misma manera, los procesos de disputa por recursos, significados, control y legitimidad dan cuenta de los territorios como construcción social, cambiante y en continuidad (Lefebvre, 2011; Ramírez, 2004; Long, 2007). En contraposición a concepciones tales como el carácter depredador del pescador artesanal, ésta perspectiva muestra el modo en que un actor elabora estrategias y utiliza conocimientos. En este sentido, la visión de la Zona Contigua como arena política deja abierta la puerta a reflexiones para una futura descentralización territorial y administrativa. Considerando lo anterior, el uso de la etnografía es útil para la construcción de verosímiles (Maidana, 2013) que describen campos relacionales a partir de la observación de juegos de intereses. En concordancia con Zhao et al. (2015), y en el intento de poner en valor una mirada integral y actual en torno a los conflictos en la pesca artesanal y las formas de disminuir sus impactos, el carácter constructivo y cotidiano de las relaciones sociopolíticas en la Zona Contigua y su Mesa Público-Privada permite avanzar desde una figura de administración a ciertas reflexiones sobre la pesca artesanal y sus relaciones con los actores del sector económico. A su vez, el enfoque centrado en el actor complementado con estrategias de triangulación de fuentes permite evidenciar la polifonía en torno a políticas públicas y de administración. Esta estrategia de investigación permite concebir escenarios sobre lo que pueden generar dichas relaciones incorporando la incertidumbre a las respuestas que desarrollen. Se trata de un avance hacia una gestión flexible y ajustable a fenómenos coyunturales y con actores territoriales con intereses diversos.

\section{BIBLIOGRAFÍA}

Abélès, M. (1997). El lugar de la política. International Social Journal, No 153: Antropología - Temas y Perspectivas: I. más allá de las lindes tradicionales.

Abélès, M. (1997). La antropología política: nuevos objetivos, nuevos objetos. Revista Internacional de Ciencias Sociales, 51-72.

Abélès, M. (2008). Política de la supervivencia. Buenos Aires: Eudeba.

Alcalá, G. (1999). Con el Agua hasta los Aparejos: Pescadores y Pesquerías en el Soconusco, Chiapas: esbozo de una historia, En J. Aréchiga, M. H., Ruz, A. B. Pérez C. et al. (Eds.). Antropología e Interdisciplina, segundo volumen de las memorias de la XXIII Mesa redonda Homenaje a Pedro Carrasco, Sociedad Mexicana de Antropología, producción de la Sociedad Mexicana de Antropología y el Instituto de Investigaciones Antropológicas de la UMAN. y Estudios Superiores en Antropología Social, Chiapas. 
Alcalá, G. (2003). Políticas pesqueras en México (19462000). Contradicciones y aciertos en la planificación de la pesca nacional. México: COLMEX-CICESECOLMICH.

Álvarez, R. (2002). Reflexiones en torno a las identidades de las poblaciones canoeras situadas entre los 44 y 48 de latitud sur, denominadas Chonos. Anales del Instituto de la Patagonia, serie Ciencias Humanas, 30, 79-86.

Andrade, B., Arenas F., \& Guijón, R. (2008). Revisión crítica del marco institucional y legal chileno de ordenamiento territorial: el caso de la zona costera. Revista de Geografía Norte Grande, 41, 23-48.

Arthur, R. I. (2005). Developing, implementing and evaluating policies to support fisheries comanagement. MRAG Ltd, London.

Aspillaga, E., Castro, M., Rodriguez, M., \& Ocampo, C. (2006). Paleopatología y estilo de vida: el ejemplo de los Chono. Revista Magallania, 34, 77-85.

Bahamonde, J. (2006). Funciones en el Diario de Viaje i Navegación del padre García. Revista Estudios Filológicos, 41, 19-30.

Baliero, W., Biasco, E., Conde, D., Cortazzo, R., Fossati, M., Gorfinkiel, D., Lorenzo, E., Menafra, R., Píriz, C., \& Roche, I. (2006). Estudio de Base sobre el Estado del Manejo Costero Integrado en Uruguay: práctica, capacitación e investigación. Proyecto Sustentabilidad de la Zona Costera Uruguaya (AUCCCIDA). Universidad de la República, Montevideo/Dalhousie University.

Barahona, N., Orensanz, J. M., Parma, A. M., Jerez, G., Romero, C., Miranda, H., Zuleta, A.,...Galvez, P. (2003). Bases Biológicas para rotación de áreas en el recurso erizo. IFOP, FIP 2000-18. Valparaíso.

Barber, M. (2010). Coastal conflicts and reciprocal relations: Encounters between Yolngu people and commercial fishermen in Blue Mud Bay, north-east Arnhem Land. Australian Journal of Anthropology, 21, 298-314.

Bárcena, A., Prado, A., Samaniego, J., \& Pérez, R. (2012). La sostenibilidad del Desarrollo. A 20 años de la cumbre para la tierra. Avances, brechas $y$ lineamientos estratégicos para América Latina y el Caribe. Naciones Unidas/Río+20.

Barragán, R., \& Wanderley, F. (2009). Etnografías del Estado en América Latina. Íconos, Revista de Ciencias Sociales. Número, 34, 21-25.

Berkes, F., \& Carlsson, L. (2004). Co-management: concepts and methodological implications. Journal of Enviromental Management, 75, 65-76.

Berkes, F. (2006). From Community-Based Resource Management to Complex Systems. Ecology and
Society, 11(1), 45.

Berkes, F., Mahon, R., Macconney, P., Pollnac, R., \& Pomeroy, R. (2001). Managing smallscale fisheries: alternative directions and methods. International Development Research Centre, Canada.

Bravo, J. M. (2004). La cultura chilota y su expresión territorial en el contexto de la globalización de la economía. Memoria para optar al Título Profesional de Geógrafo, Universidad de Chile, Escuela de Geografía, Santiago.

Bremer, S., \& Glavovic, B. (2013). Mobilizing Knowledge for Coastal Governance: Re-Framing the Science-Policy Interface for Integrated Coastal Management. Journal Coastal Management, 41(1), 39-56.

Bugueño, Z. (2011). Gobernanza y Uso del Territorio en Localidades Litorales Caso de estudio: San Juan y Tenaún, Comuna de Dalcahue, X Región de Los Lagos. Memoria para optar al título de Geógrafo. Universidad de Chile.

Bullimore, B. (2014). Problems and pressures, management and measures in a site of marine conservation importance: Carmarthen Bay and Estuaries. Estuarine, Coastal and Shelf Science, 150, 288-298.

CEPAL/PNUMA. (2012). La sostenibilidad del desarrollo en América Latina y el Caribe: Desafíos y oportunidades. Comisión Económica para América Latina y el Caribe/ Oficina Regional para América Latina y el Caribe. Santiago de Chile.

Cordero, E. (2011). Ordenamiento territorial, justicia ambiental y zonas costeras. Revista de derecho (Valparaíso), 36, 209-249.

Crona, B., \& Bodin, Ö. (2009). Social Networks and Leadership in Natural Resources Governance: are the important and how can we study them? Stockholm Resilience Center, Stockholm niversity, Stockholm, Sweden. Conference at the 7th International Science Conference on the Human Dimensions of Global Environmental Change. International Human Dimensions Programme Open Meeting. The Social Challenges of Global Change.

Cundill, G. (2010). Monitoring Social Learning Processes in Adaptive Co-management: Three Case Stuidies from South Africa. Ecology and Society, 15(3), 28.

Chirif, A. (2008). Prólogo: El manejo de recursos como práctica democrática. En D. Pinedo \& C. Soria (Eds.), El manejo de las pesquerías en los ríos tropicales de Sudamérica. Mayol Ediciones S. A. Bogotá, Colombia.

De la Maza, F. (2009). Etnografía del gobierno local: contingencias políticas y prácticas sociales en dos comunas de la Región de la Araucanía y los Ríos, Chile. 
Pontificia Universidad Católica de Chile. VIII Reunión de Antropología del Mercosur.

De la Maza, F. (2012). Etnografía de las prácticas sociales y negociaciones en la política indígena actual, AraucaníaChile. Rev. Austral Cienc. Soc. 22, 85-100.

Devlin, R, \& Moguillansky, G. (2009). Alianzas público-privadas como estrategias nacionales de desarrollo a largo plazo. Revista CEPAL, 97, 97-116.

Díaz, A. (2008) Procesos Sociales y Lógicas Productivas en la Relación entre Pescadores Artesanales, Estado y Mercado. El caso de las organizaciones de pescadores artesanales de la Comuna de Corral, Región de los Ríos. Tesis de Grado para optar al Título de Antropóloga. Universidad Austral de Chile.

Duhart, H. (2004). ¿Constituye la regulación actual una herramienta eficaz para la explotación sustentable del recurso pesquero?. Memoria de prueba para optar al grado de Licenciado en Ciencias Jurídicas y Sociales. Universidad Austral de Chile. Facultad de Ciencias Jurídicas y Sociales. Escuela de Derecho.

Emperaire, J. (1963). Los Nómades del mar. Universidad de Chile. Santiago de Chile.

Foucault, M. (2001). Dits et écrits I y II. Paris: Gallimard.

Ghermandi, A., Nunes, P., Portela, R., Nalini, R., \& Sonja, T. (2010). Recreational, Cultural, and Aesthetic Services from Estuarine and Coastal Ecosystems. Reference Module in Earth Systems and Environmental Sciences, Treatise on Estuarine and Coastal Science, 12, 217. 237.

Gelcich, S., Edwards-Jones, E., Kaiser, M., \& Castilla, J. C. (2008). Comanagement Policy Can Reduce Resilience in Traditionally Managed. Marine Ecosystems, 9(6), 951-966.

Gelcich S., Hughes, T. P., Olsson, P., Folke C., Defeo O., Fernández M., Foale S.,...Castilla JC. (2010). Navigating transformations in governance of Chilean marine coastal resources. PNAS (Proceedings of the National Academy of Sciences of the United States of America) 107, 16794-16799.

Gómez, J. (2003). Subjetividades políticas: desafíos y debates latinoamericanos. Compiladores Claudia Pidrahita, Álvaro Díaz y Pablo Vommaro. $1^{\text {a }}$ ed. Bogotá. Universidad Distrital Francisco José de Caldas

Guerra, D., \& Skewes, J. C. (2008). ¿Vernacularización, hibridación, enajenación o patrimonialización? Disyuntivas locales en la construcción del paisaje. Conserva, 12, 5-37.

Gutiérrez, G., Matamala, M., \& Henríquez, J. (2006). Bases para el plan de manejo pesquerías bentónicas Zona Contigua Región X y XI. Consultora Pupelede.
IFOP (2012). Recursos Objetivo Areas de Manejo Pesca Artesanal - Chile. Erizo Loxechinus Albus. Disponible en: http://www.ifop.cl/wp-content/uploads/recursos_ amerb/Erizo_2012.pdf

Hevia, F. (2009). Relaciones sociedad-Estado: análisis interactivo para una antropología del Estado. Espiral, Estudios sobre Estado y Sociedad, XV, 45, 43-70.

Hidalgo, C. (2011). La práctica pesquero artesanal y su vínculo con la expansión de la economía de capitales: aproximación antropológica a la pesca artesanal en las caletas de Islas Huichas, Puerto Cisnes y Puerto Melinka, Región de Aysén del General Carlos Ibáñez del Campo. Temuco: Tesis para optar al grado de licenciado en Antropología. Departamento de Antropología. Facultad de Ciencias Sociales y Jurídicas. Universidad Católica de Temuco

Hucke-Gaete, R; Álvarez, R; Ruiz, J., \& Torres, J. P. (2008) Investigación para el desarrollo del área marina costera protegida de Chiloé. Primer Informe de avance. Proyecto BIP N ${ }^{\circ}$ 30040215-0. Universidad austral de Chile.

Hucke-Gaete, R., Lo Moro, P., \& Ruiz, J. (2010). Síntesis del estudio Investigación para el desarrollo de Área Marina Costera Protegida Chiloé, Palena y Guaitecas. Gore los Lagos/MMA/Uach.

Instituto de Fomento Pesquero (IFOP). (2012). Ficha Erizo (Loxechinus albus). Recurso Objetivo Áreas de Manejo Pesca Artesanal - Chile

Keene, S., Gibrán, Y., \& Stotz, W. (2002). Competing Visions for Marine Tenure and Co-Management: Genesis of a Marine Management Area System in Chile. Coastal Management, 30, 85-99.

Labra, G. (2011). Distribución Espacial de Quistes de Alexandrium Catenella Y Protoceratium Reticulatum en dos Sectores del Litoral Norte de la Región de Aysén, Archipielago de las Guaitecas y Estero Pitipalena. Tesis para Optar al Título de: Ingeniero en Acuicultura. Universidad Austral de Chile.

Larsen, R. K., Acebes, J. M., \& Belen, A. (2011). Examining the assumptions of integrated coastal management: Stakeholder agendas and elite cooption in Babuyan Islands, Philippines. Journal Ocean \& Coastal Management, 54(1), 10-18.

Leff, E. (1996). La capitalización de la naturaleza y las estrategias fatales de la sustentabilidad. Boletín de la Red Ambiental, 7(16), 17-20.

Legoupil, D. (2005). Recolectores de moluscos tempranos en el sureste de la isla de Chiloé: Una primera mirada. Magallania, 33(1), 51-61.

Lefebure, Henri (1976). Espacio y política: el derecho a la 
ciudad II. Barcelona: Ediciones Península.

Lockwood, M., \& Davidson, J. (2010). Environmental governance and the hybrid regime of Australian natural resource management. Journal Geoforum, 41, 388398.

Long, N. (2007). Sociología del Desarrollo: una perspectiva centrada en el actor. México: CIESAS.

Maidana, C. (2013). Migración indígena, procesos de territorialización y análisis de redes sociales. REMHU - Revista Interdisciplinar da Mobilidade Humana, Brasília, Ano XXI, n. 41, p. 277-293, jul./dez.A.

Marin, A., \& Berkes, F. (2010). Network approach for understanding small-scale fisheries governance: The case of the Chilean coastal co-management system. Marine Policy, 34(5), 851-858.

Marin, A., \& Gelcich, S. (2012). Gobernanza y capital social en el comanejo de recursos bentónicos en Chile: Aportes de redes al estudio de la pesca artesanal de pequeña escala. CURSHO. Cultural-Hombre-Sociedad, 1,131153.

Marticorena, L. (2009). A pura memoria: Conocimientos y significados de la naturaleza en las localidades de Melinka y Repollal, Litoral norte de la Región de Aysén. Valdivia: Tesis para optar al titulo de licenciada en Antropología, Antropóloga. Escuela de Antropología. Facultad de Filosofía y Humanidades. Universidad Austral de Chile.

Martínez-Alier, J. (2004). Los Conflictos Ecológico-Distributivos y los Indicadores de Sustentabilidad. Revista Iberoamericana de Economía Ecológica, 1, 21-30

Martínez-Alier, J. (2011). El Ecologismo de los pobres: conflictos ambientales y lenguajes de valoración. Nueva edición aumentada y publicada en Perú por Espiritrompa Ediciones y en Barcelona por Editorial Icaria.

Martínez, V.(2012). Uso del espacio marítimo de los pueblos originarios y de los recursos hidrobiológicos. Efectos jurídicos. Memoria para optar al grado de Licenciado en Ciencias Políticas Jurídicas y Sociales. Universidad Austral de Chile.

Martinic, M. (2005). De la Trapananda al Aysén: una mirada reflexiva sobre el acontecer de la Región de Aysén desde la prehistoria hasta nuestros dias. Santiago: Pehuén Editores.

Medina, M. (1997). Estudio comparativo del fitoplancton del seno Aysén, XI región, y variables oceanográficas asociadas en cruceros de verano y otoño (1992). Tesis, Carrera de Biología Marina. Facultad de Ciencias del Mar. Universidad de Valparaíso.
Moreno, C., Barahona, N., Molinet, C., Orensanz, J. M., Parma

A. M., \& Zuleta, A. (2007). From crisis to institutional sustainability in the Chilean sea urchin fishery. En T. R. McClanahan \& J. C. Castilla (Eds.). Fisheries Management: Progress towards Sustainability (pp. 43-65). Blackwell, Publ.

Molinet, C., Fuentealba M., Arévalo A., Barahona, N., Asencio C., Ariz L., González G.,...Paredes C. (2007). Diagnóstico biológico-pesquero para recursos bentónicos de la Zona Contigua, X y XI Región. Informe Final. Proyecto FIP N²005-51. Universidad Austral de Chile. Entidad ejecutora.

Montecinos, E. (2007). Los límites de la modernización municipal para profundizar la participación ciudadana en Chile ¿Es la gobernanza de proximidad una alternativa? Gestión y Organización, XVI(2), 319-351.

Munizaga, C. (1988). Antropología Cultural”, en Chiloé y su influjo en la XI región, II Jornadas Territoriales, Colección Terra Nostra, $n^{\circ} 12$. Santiago.

Muñoz, P., Avaria, S., Sievers, H., \& Prado, R. (1992). Presencia de Dinoflagelados tóxicos del género Dinophysis en el seno Aysén, Chile. Revista de Biología Marina, 27(2), 187-212.

Navarro, M. (2008). Comunidades Humanas y Poblaciones de Grandes Ballenas: Una aproximación desde la Antropología al patrimonio natural y cultural de las localidades del Archipiélago de los Chonos, Región de Aysén, Chile. Valdivia: Tesis de Grado para optar al título de Antropólogo y al grado de Licenciado en Antropología. Universidad Austral de Chile.

Nuñez, N. (2010). La transformación del trabajo como práctica económico cultural en comunidades de pescadores artesanales: nuevos significados a partir de la introducción y expansión de la industria salmonera. El caso de la comuna de Guaitecas, Región de Aysén. Temuco: Tesis para optar al Grado de Licenciado en Antropología. Departamento de Antropología. Facultad de Ciencias Sociales y Jurídicas. Universidad Católica de Temuco.

Olguin, A., \& Barahona, N. (2007). Análisis de la pesquería de jaiba en los principales centros de desembarque a nivel regional. Seguimiento del Estado de Situación de las Principales pesquerías Nacionales: Proyecto Investigación Situación Pesquerías Bentónicas, 2006. IFOP - Subpesca, Informe Técnico $\mathrm{N}^{\circ} 3$

Olsson, P., Folke, C., \& Berkes, F. (2004a). Adaptive CoManagement for Building Resilience in Social-Ecological Systems. Environmental Management, 34(1), 75-90.

Olsson, P., Folke, C., \& Hahn, T. (2004b). Social-ecological transformation for ecosystem management: the 
development of adaptive co-management of a wetland landscape in southern Sweden. Ecology and Society 9(4), 2.

Pamplona, F. (2000). Sustentabilidad y políticas públicas. Gaceta Ecológica, 56, 46-53.

Pickard, G. L. (1971). Some physical oceanographic feature of inlets of Chile. Journal Fisheries Research Board of Canada, 28, 1077-1106.

Saavedra, G. (2007). Las economías silenciosas del litoral aisenino. En M. Osorio (Ed.). Otras narrativas en Patagonia. Tres miradas antropológicas a la Región de Aisén, (pp. 35-63). Coyhaique, Ñirre Negro.

Saavedra, G. (2008). Desarrollo Endógeno y Dinámicas de Significación Cultural en el Borde Costero del Sur Austral Chileno. Simposio Antropoloxía e Cooperación ao Desenvolvemento (I Simposio de ALGA, Asociación Luso-Galega de Antropoloxía Aplicada).

Saavedra, G. (2010). Tradición e innovación en las comunidades de pesca artesanal del sur de chile: hacia un enfoque reflexivo del desarrollo endógeno. AIBR. Revista de Antropología Iberoamericana, 7(1), 33-63,

Saavedra, G. (2011a). Desarrollo, subjetividad y transgresiones identitarias en las costas del sur- austral chileno. Sociedad \& Equidad, 2, 282-303.

Saavedra, G. (2011b). Perspectivas culturales del desarrollo en las costas australes de Chile. Aproximación antropológica a las persistencias y transformaciones de las economías de pesca artesanal en el litoral de Aisén. Memoria para optar al grado de doctor. Facultad de Ciencias Políticas y Sociología. Departamento de ciencia política y de la administración. Universidad Complutense de Madrid

Saavedra, G. (2013). La pesca artesanal en las encrucijadas de la modernización. Usos, apropiaciones y conflictos en el borde costero del sur de Chile. Revista andaluza de antropología, 4, 79-102.

Schaper, M. (1995). Comercio internacional y medio ambiente. Una perspectiva económica En El Desarrollo Sostenible en la economía de América Latina, CECODES, CLADEI. Santa Fe de Bogotá.

Schavelzon, S. (2008). Antropología del Estado en Bolivia: Verdades Sagradas, Farsas Políticas y Definiciones de
Identidad. Cuadernos de Antropología, Sección de Antropología Social, 28, 67-84.

Silva, N., Sievers, H. A., \& Prado, R. (1958). Características oceanográficas y una proposición de circulación, para algunos canales australes de Chile entre $41^{\circ} 20^{\prime} \mathrm{S}, 46^{\circ}$ 40' S. Revista de Biología Marina, 30(2), 207-254.

Skewes, J. C., Álvarez, R., \& Navarro, M. (2012). Usos consuetudinarios, conflictos actuales y conservación en el borde costero de Chiloé insular. Magallania, 40(1), 109-125.

Solari, M., Skewes, J., Navarro, M., \& Fabían P. (2012). Historia ambiental de los archipiélagos de la Trapananda (Patagonia septentrional, Chile): desafíos para la conservación de la ballena azul. CUHSO. CulturaHombre-Sociedad, 22(1), 115-130

Subpesca. (2012). Cuota de captura recurso erizo (Loxechinus albus) Regiones X y XI. Informe Técnico (R. Pesq.) $\mathrm{N}^{\circ}$ 24-2012.

Subsecretaría de Pesca (2011). Informe Técnico (R.Pesq.) $N^{\circ}$ 071-2011. Disminución Talla Mínima Legal del Recurso Erizo (Loxechinus albus, Molina) Regiones X y XI.

Ther Ríos, F. (2011). Configuraciones del tiempo en el mar interior de Chiloé y su relación con la apropiación de los territorios marítimos. Desenvolvimiento $y$ medioambiente, $23,67-80$.

Torres, A., \& Becerra, A. (2006). La construcción del objeto y los referentes teóricos en la investigación social. En A. Becerra \& A. Torres (Comp.). La práctica investigativa en ciencias sociales. (pp. 15-29). DCS, Departamento de Ciencias Sociales. UPN, Universidad Pedagógica Nacional, Bogotá.

Wasson, K., Suarez, B., Akhavan, A., McCarthy, E., Kildow, J., Johnson, K., Fountain, M., Woolfolk, A.,...Feliz, D. (2015). Lessons learned from an ecosystem-based management approach to restoration of a California estuary. Marine Policy, 58, 60-70.

Zhao, M., Yang, D., Wang, P., \& Ping, S. (2015). A marketbased approach to marine sand resource management in the Pearl River estuary, China. Journal Ocean \& Coastal Management, 105, 56-64. 
